\title{
Spectral Properties of Partial Integral Operators of Volterra and Volterra-Fredholm Type
}

\author{
A. S. Kalitvin
}

\begin{abstract}
We give sufficient conditions under which the spectral radius of a partial integral operator of Volterra type is zero, or a partial integral operator of Volterra-Fredholm type has a trivial essential spectrum.
\end{abstract}

Keywords: Partial integral operators, Volterra operators, Volterra-Fredholm operators, spectral radius, essential spectrum

AMS subject classification: 47 G 99, 46 E 30, 47 A 10, 47 A 25, 47 A 53

\section{Introduction}

Let $a, b>0$, and let $\Omega$ denote either $[0, t] \times[0, s],[0, t] \times[0, b]$ or $[0, a] \times[0, s]$. Given measurable functions

$$
l=l(t, s, \tau), \quad m=m(t, s, \sigma), \quad n=n(t, s, \tau, \sigma)
$$

consider the linear partial integral operator of Volterra type

$$
\begin{aligned}
(K x)(t, s)= & \int_{0}^{t} l(t, s, \tau) x(\tau, s) d \tau \\
& +\int_{0}^{s} m(t, s, \sigma) x(t, \sigma) d \sigma \quad(0 \leq t \leq a, 0 \leq s \leq b) . \\
& +\int_{\Omega} n(t, s, \tau, \sigma) x(\tau, \sigma) d(\tau, \sigma)
\end{aligned}
$$

In what follows, we denote the operators which are defined by the first, second and third integral in (1) by $L, M$ and $N$, respectively. Further, by $P_{D}$ we denote the multiplication operator by the characteristic function $\chi_{D}$ of some measurable set $D$, and by $S=S(\Delta)$ the space of all (real or complex) functions on a measurable set $\Delta$ which are measurable and almost everywhere finite. Recall $[7,11]$ that a Banach space $Z \subset S$ is called ideal space if the relations $x \in S, z \in Z$ and $|x(\omega)| \leq|z(\omega)|$ a.e. on $\Delta$ imply that also $x \in Z$

A. S. Kalitvin: Pedag. Inst., Dept. Math., ul. Lenina 42, R-398020 Lipetsk, Russia e-mail: kas@pedinst.lipetsk.su 
and $\|x\| \leq\|z\|$. An ideal space $Z$ is called regular if $\left\|P_{D} z\right\| \rightarrow 0$ as mes $D \rightarrow 0$ for each $z \in Z$, and almost perfect if $\|z\| \leq \lim _{n \rightarrow \infty}\left\|z_{n}\right\|$ for each sequence $z_{n}$ in $Z$ which converges in measure to $z \in Z$. Examples of almost perfect spaces are the Lebesgue space $L^{p} \quad(1 \leq p \leq \infty)$ and Orlicz spaces; for $1 \leq p<\infty$ the space $L^{p}$ is regular.

Suppose that the operator (1) is bounded in some ideal space $U$ of measurable functions on $[0, a] \times[0, b]$. Then the operator

$$
P_{[c, \bar{c}] \times[d, \bar{d}]} K P_{[c, \bar{c}] \times[d, \bar{d}]} \quad(0 \leq c \leq \tilde{c} \leq a, 0 \leq d \leq \tilde{d} \leq b)
$$

is also bounded in $U$. Denote by $r(K)$ the spectral radius of the operator $K$, and let

$$
\delta(K)=\varlimsup_{\substack{\tilde{c}-c \rightarrow 0 \\ \bar{d}-d \rightarrow 0}}\left\|P_{[c, \bar{c}] \times[d, \bar{d}]} K P_{[c, \bar{c}] \times[d, \bar{d}]}\right\| .
$$

Consider the operator ( 1 ) first with $\Omega=[0, t] \times[0, s]$. The following result may be found in [12].

Theorem 1. If the operator $K$ is bounded in the space $U$, then the estimate

$$
r(K) \leq \delta(K)
$$

is true.

The following example shows that one may have strict inequality in (3).

Example 1. Let $a=b=1, m(t, s, \sigma)=n(t, s, \tau, \sigma)=0$, and

$$
l(t, s, \tau)=\bar{l}(t, \tau)= \begin{cases}2^{n} & \text { if } 2^{-n} \leq \tau \leq t<2^{1-n} \\ 0 & \text { otherwise. }\end{cases}
$$

The operator

$$
\left(\bar{L}_{x}\right)(t)=\int_{0}^{t} \bar{l}(t, \tau) x(\tau) d \tau
$$

acts in $L^{\infty}([0,1])$ with $r(\bar{L})=0 \quad[10]$. Consequently, the corresponding operator (1) acts in $L^{\infty}([0,1])$ with $r(K)=0$. On the other hand, choosing $x(t, s) \equiv 1$ we see that the function $P_{[c, \bar{c}] \times[d, \tilde{d}]} K P_{[c, \tilde{c}] \times[d, \bar{d}]} x$ takes the value 1 on a set of positive measure, and thus $\delta(K)=1$.

Observe that the number (2) satisfies the properties

$$
\delta(K) \geq 0, \quad \delta\left(K_{1}+K_{2}\right) \leq \delta\left(K_{1}\right)+\sigma\left(K_{2}\right), \quad \delta(\lambda K)=|\lambda| \delta(K)
$$

on the space $\mathcal{L}_{W}(U, U)$ of all operators of the form (1). We say that the operator (1) has the Ando property if

$$
\lim _{\substack{\operatorname{mes} D_{1} \rightarrow 0 \\ \text { mes } D_{2} \rightarrow 0}}\left\|P_{D_{1} \times D_{2}} K P_{D_{1} \times D_{2}}\right\|=0
$$


Of course, the Andô property holds for the operator $K$ above if it holds for the components $L ; M$ and $N$, and may be verified by the usual majorant techniques (see, e.g., [5, $6]$ ). In particular, if the operator $|K|$ defined by

$$
\begin{array}{rlr}
(|K| x)(t, s)= & \int_{0}^{t}|l(t, s, \tau)| x(\tau, s) d \tau \\
& +\int_{0}^{s}|m(t, s, \sigma)| x(t, \sigma) d \sigma \quad(0 \leq t \leq a, 0 \leq s \leq b) \\
& +\int_{\Omega}|n(t, s, \tau, \sigma)| x(\tau, \sigma) d(\tau, \sigma)
\end{array}
$$

has the Andô property, then the operator (1) has of course the Andô property as well. In [5] it was shown that the operator (5) is bounded in an ideal space $U$ if and only if the operators $|L|,|M|$ and $|N|$ defined by the first, second and third integral in (5) are bounded in $U$. Consequently, the Ando property holds for the operator $|K|$ if and only if it holds for the components $|L|,|M|$, and $|N|$. We remark that the operator (1) is regular in an ideal space $U$ (i.e. may be majorized by a positive operator in $U$ ) if and only if the operator (5) acts in $U$ [5].

We point out that our definition of the Andô property is somewhat different from that given in $[6,12]$, where (4) is replaced by

$$
\lim _{\operatorname{mes} \rightarrow 0}\left\|P_{D} A P_{D}\right\|=0
$$

but $D$ is not necessarily a "rectangle" $D_{1} \times D_{2}$ with mes $D_{1} \rightarrow 0$ and mes $D_{2} \rightarrow 0$; this is a stronger condition than ours. For example, the operator $K$ with constant kernels has the Andô property in $L^{\infty}([0, a] \times[0, b])$ in our sense, but not in the sense of [12].

The following is a straightforward consequence of Theorem 1.

Theorem 2. If the operator $K$ is bounded in the space $U$ and has the Ando property, then $\delta(K)=r(K)=0$.

The example of the partial integral operator of Hardy-Littlewood type

$$
(K x)(t, s)=\frac{1}{t} \int_{0}^{t} x(\tau, s) d \tau+\frac{1}{s} \int_{0}^{s} x(t, \sigma) d \sigma
$$

in $U=L_{p}([0,1] \times[0,1])(1<p<\infty)$ shows that the spectral radius of a partial integral operator of Volterra type may be strictly positive. Thus, the equality $r(K)=0$ is not true in general.

For $0 \leq s \leq b$ and $0 \leq t \leq a$ let

$$
L(s) x(t)=\int_{0}^{t} l(t, s, \tau) x(\tau) d \tau, \quad|L(s)| x(t)=\int_{0}^{t}|l(t, s, \tau)| x(\tau) d \tau
$$


and

$$
M(t) y(s)=\int_{0}^{s} m(t, s, \sigma) y(\sigma) d \sigma, \quad|M(t)| y(s)=\int_{0}^{s}|m(t, s, \sigma)| y(\sigma) d \sigma
$$

If the operators $L(s)(0 \leq s \leq b)$ and $M(t)(0 \leq t \leq a)$ are regular and compact in two ideal spaces $X$ and $Y$, respectively, then the resolvent kernels $\phi(t, s, \tau)$ and $\psi(t, s, \sigma)$ of these operators may be represented as series of iterated kernels

$$
\phi(t, s, \tau)=\sum_{k=1}^{\infty} \lambda^{-k} l^{(k)}(t, s, \tau)
$$

and

$$
\psi(t, s, \sigma)=\sum_{k=1}^{\infty} \lambda^{-k} m^{(k)}(t, s, \sigma),
$$

which converge in the so-called Zaanen spaces $\mathcal{Z}(X, X)$ and $\mathcal{Z}(Y, Y)$, respectively $[6,9]$. We remark that the spaces $\mathcal{Z}(X, X)$ and $\mathcal{Z}(Y, Y)$ consist of all kernels which generate regular integral operators in $X$ and $Y$, respectively.

To state our next theorem, another definition is in order. Let $X$ be an almost perfect ideal space over $[0, a]$, and $Y$ an almost perfect ideal space over $[0, b]$. The space with mixed norm $Y[X]$ consists, by definition, of all measurable functions $x$ on $[0, a] \times[0, b]$ for which the norm

$$
\|x\|_{Y[X]}=\|s \mapsto\| x(\cdot, s)\left\|_{X}\right\|_{Y}
$$

is finite. Similarly, the space with mixed norm $X[Y]$ is defined by the norm

$$
\|x\|_{X[Y]}=\|t \mapsto\| x(t, \cdot)\left\|_{Y}\right\|_{X} .
$$

Important results on ideal spaces with mixed norm may be found in [3]. In particular, the spaces $Y[X]$ and $X[Y]$ are regular (resp. almost perfect) if the spaces $X$ and $Y$ are.

\section{Results for Volterra operators}

Let $U$ be an ideal space over $[0, a] \times[0, b]$. By $\mathcal{R}_{l}(U, U)$ and $\mathcal{R}_{m}(U, U)$ we denote the sets of all measurable functions $l=l(t, s, \tau)$ on $[0, a] \times[0, b] \times[0, a]$ and $m=m(t, s, \sigma)$ on $[0, a] \times[0, b] \times[0, b]$, respectively, with

$$
\|l\|_{\mathcal{R}_{1}(U, U)}=\sup _{\|x\| U \leq 1}\left\|\int_{0}^{a}|l(\cdot, \cdot, \tau) x(\tau, \cdot)| d \tau\right\|_{U}<\infty
$$

and

$$
\|m\|_{\mathcal{R}_{m}(U, U)}=\sup _{\|x\|_{U} \leq 1}\left\|\int_{0}^{b}|m(\cdot, \cdot, \sigma) x(\cdot, \sigma)| d \sigma\right\|_{U}<\infty .
$$

These spaces have been studied in [5]. 
Theorem 3. Let $X$ and $Y$ be almost perfect ideal spaces over $[0, a]$ and $[0, b]$, respectively, and $U=Y[X]$ or $U=X[Y]$. Suppose that the operator (1) is regular in $U$, and the operators $|L(s)|(s \in[0, b]),|M(t)|(t \in[0, a])$ and $N$ are compact in $X, Y$ and $U$, respectively. Finally, assume that the resolvent kernels $\phi(t, s, \tau)$ and $\psi(t, s, \sigma)$ belongs to $\mathcal{R}_{l}(U, U)$ and $\mathcal{R}_{m}(U, U)$, respectively, and at least one of the operators $L M$ or $M L$ is compact in $U$. Then the spectral radius of the operator (1) is zero.

Proof. It suffices to show that, for any $f \in U$ and every complex number $\mu=\lambda^{-1}$, the equation $x=\mu K x+f$ has a unique solution $x \in U$. Since $X$ is an ideal space and the operators $|L(s)|(s \in[0, b])$ are compact in $X$, we have $r(|L(s)|)=0$ for all $s \in[0, b]$ [8]. Analogously, $r(|M(t)|)=0$ for all $t \in[0, a]$. This means that $\phi(t, s, \tau)$ and $\psi(t, s, \sigma)$ are limits of the series (9) and (10) which converge in the Zaanen spaces $\mathcal{Z}(X, X)$ and $\mathcal{Z}(Y, Y)$. From this and the hypotheses $\phi \in \mathcal{R}_{l}(U, U)$ and $\psi \in \mathcal{R}_{m}(U, U)$ it follows that the operators

$$
\Phi x(t, s)=x(t, s)+\int_{0}^{t} \phi(t, s, \tau) x(\tau, s) d \tau
$$

and

$$
\Psi x(t, s)=x(t, s)+\int_{0}^{s} \psi(t, s, \sigma) x(t, \sigma) d \sigma
$$

are inverse, respectively, to the operators $I-\mu L$ and $I-\mu M$ in the space $U$. But this shows that the equation $x=\mu K x+f$, with $f \in U$, is equivalent to the Volterra equations

$$
\begin{aligned}
& {\left[I-(I-\mu M)^{-1}(I-\mu L)^{-1} \mu(N+\mu L M)\right] x=g} \\
& {\left[I-(I-\mu L)^{-1}(I-\mu M)^{-1} \mu(N+\mu M L)\right] x=h}
\end{aligned}
$$

where

$$
g:=(I-\mu M)^{-1}(I-\mu L)^{-1} f \quad \text { and } \quad h:=(I-\mu L)^{-1}(I-\mu M)^{-1} f
$$

At least one of these equations contains a compact operator in an ideal space, and hence has a unique solution. This shows that the spectral radius of the operator (1) is zero as claimed

The hypotheses of Theorem 3 are rather easy to verify in the case $X=L^{p}([0, a])$ and $Y=L^{p}([0, b]) \quad(1 \leq p \leq \infty)$. Here the conditions $\phi \in \mathcal{R}_{l}(U, U)$ and $\psi \in \mathcal{R}_{m}(U, U)$ mean that the partial integral operators defined by the kernels $\phi(t, s, \tau)$ and $\psi(t, s, \sigma)$ are regular in $U$. The following technique for estimating $\delta(L)$ and $\delta(M)$ is taken from [12].

Suppose that the operator $L(s)$ acts, for each $s \in[0, b]$, in an ideal space $X$ over $[0, a]$, and the operator $M(t)$ acts, for each $t \in[0, a]$, in an ideal space $Y$ over $[0, b]$. Let

$$
\alpha(s)=\|L(s)\|_{X \rightarrow X} \quad \text { and } \quad \beta(t)=\|M(t)\|_{Y \rightarrow Y}
$$

Then the operator $L$ acts in $Y[X]$ if $\alpha \in L^{\infty}$, and the operator $M$ acts in $X[Y]$ if $\beta \in L^{\infty} ;$ in this case, $\|L\| \leq\|\alpha\|_{L^{\infty}}$ and $\|M\| \leq\|\beta\|_{L^{\infty}}$ [5]. From (2) and (14) we get the estimates

$$
\delta(L) \leq \varlimsup_{\overline{\lim }}{ }_{\tilde{c}-c \rightarrow 0}\|s \mapsto\| P_{[c, \tilde{c}]} L(s) P_{[c, \tilde{c}]}\left\|_{X \rightarrow X}\right\|_{L^{\infty}}
$$


and

$$
\delta(M) \leq \overline{\lim }_{\bar{d}-d \rightarrow 0}\|t \mapsto\| P_{[d, \bar{d}]} M(t) P_{[d, \bar{d}]}\left\|_{Y \rightarrow Y}\right\|_{L^{\infty}}
$$

Now, if the operators $L(s)$ and $M(t)$ from (7) and (8) have the Andô property, i.e.

$$
\lim _{\operatorname{mes} D \rightarrow 0} \sup _{0 \leq s \leq b}\left\|P_{D} L(s) P_{D}\right\|_{X \rightarrow X}=0
$$

and

$$
\lim _{\text {mes } D \rightarrow 0} \sup _{0 \leq t \leq a}\left\|P_{D} M(t) P_{D}\right\|_{Y \rightarrow Y}=0
$$

then $\delta(L)=r(L)=\delta(M)=r(M)=0$, where $L$ is considered as an operator in $Y[X]$, and $M$ as an operator in $X[Y]$. The relations (17) and (18) are usually proved by means of majorant techniques.

Our discussion implies the following result [12].

Theorem 4. Let $X=L^{p}([0, a])$ and $Y=L^{p}([0, b])(1 \leq p \leq \infty)$. Suppose that the linear integral operators $L(s)(0 \leq s \leq b)$ and $M(t)(0 \leq t \leq a)$ act in the spaces $X$ and $Y$, respectively, and have the Ando property. Moreover, assume that the linear integral operator $N$ acts in the space $U=L^{p}([0, a] \times[0, b])$ and has also the Ando property. Then the operator $K$ satisfies $\delta(K)=r(K)=0$ in $U$.

Let us give still another method for estimating the numbers $\delta(L)$ and $\delta(M)$. Define operators $\tilde{L}$ and $\tilde{M}$ by

$$
\tilde{L} x(t)=\int_{0}^{t} \tilde{l}(t, \tau) x(\tau) d \tau \quad \text { and } \quad \tilde{M} y(s)=\int_{0}^{s} \tilde{m}(s, \sigma) y(\sigma) d \sigma
$$

where

$$
\tilde{l}(t, \tau)=\|l(t, \cdot, \tau)\|_{L^{\infty}} \quad \text { and } \quad \tilde{m}(s, \sigma)=\|m(\cdot, s, \sigma)\|_{L^{\infty}} .
$$

If the operator $\tilde{L}$ acts in an ideal space $X$ over $[0, a]$, and the operator $\tilde{M}$ acts in an ideal space $Y$ over $[0, b]$, then the operator $L$ acts in $X[Y]$ and the operator $M$ acts in $Y[X][5]$. In this case

$$
\|L\|_{X[Y] \rightarrow X[Y]} \leq\|\| l(t, \cdot, \tau)\left\|_{L^{\infty}}\right\|_{\boldsymbol{Z}(X, X)}
$$

and

$$
\|M\|_{Y[X] \rightarrow Y[X]} \leq\|\| m(\cdot, s, \sigma)\left\|_{L^{\infty}}\right\|_{Z(Y, Y)} .
$$

These estimates imply in turn that

$$
\delta(L) \leq \varlimsup_{\tilde{\lim }-c \rightarrow 0}\left\|P_{[c, \bar{c}]} \tilde{L} P_{[c, \tilde{c}]}\right\|_{\mathcal{L}_{r}(X, X)}=\delta(\tilde{L})
$$

and

$$
\delta(M) \leq \varlimsup_{\bar{d}-d \rightarrow 0}\left\|P_{[d, \bar{d}]} \tilde{M} P_{[d, \tilde{d}]}\right\|_{\mathcal{L}_{r}(Y, Y)}=\delta(\tilde{M}),
$$

where $\mathcal{L}_{r}(Z, Z)$ denotes the space of all regular linear operators acting in $Z$. From the Ando property of the operators $\tilde{L}$ and $\tilde{M}$ it follows that $\delta(L)=\delta(\tilde{L})=0$ in $X[Y]$ and $\delta(M)=\delta(\tilde{M})=0$ in $Y[X]$. In this way, we have proved the following 
Theorem 5. Suppose that the operators $\tilde{L}, \tilde{M}$ and $N$ act in the spaces $L^{p}([0, a])$, $L^{p}([0, b])$ and $U=L^{p}([0, a] \times[0, b])$, respectively, and all have the Ando property. Then the operator (1) acts in $U$ and satisfies $\delta(K)=r(K)=0$.

Analogous results hold for the spaces $Y[X]$ and $X[Y]$ :

Theorem 6. Let $X$ and $Y$ be ideal spaces over $[0, a]$ and $[0, b]$, respectively. Suppose that the operators

a) $L(s)(0 \leq s \leq b), \tilde{M}$ and $N$ act in $X, Y$ and $Y[X]$, respectively or

b) $M(t)(0 \leq t \leq a), \tilde{L}$ and $N$ act in $Y, X$ and $X[Y]$, respectively and have the Andô property. Moreover, assume that $\alpha \in L^{\infty}$ or $\beta \in L^{\infty}$, respectively. Then the operator (1) act in $Y[X]$ or $X[Y]$, respectively, and satisfies $\delta(K)=r(K)=0$.

Proof. The inclusion $L(Y[X]) \subseteq Y[X]$ and the equality $\delta(L)=0$ follow from the inclusion $\alpha \in L^{\infty}$ and the relations (15) and (17). The definition (19) and the estimate (21) imply that $M: Y[X] \mapsto Y[X]$ and $\delta(M)=0$. Consequently, the operator (1) acts in $Y[X]$ with $0 \leq r(K) \leq \delta(K) \leq \delta(L)+\delta(M)+\delta(N)=0$

Now we are going to study the case $\Omega=[0, t] \times[0, b]$. To this end, we associate to the operator

$$
(N x)(t, s)=\int_{0}^{t} \int_{0}^{b} n(t, s, \tau, \sigma) x(\tau, \sigma) d \tau d \sigma
$$

the number

$$
\delta_{t}(N)=\overline{\lim }_{\tilde{c}-c \rightarrow 0}\left\|P_{[c, \bar{c}] \times[0, b]} N P_{[c, \bar{c}] \times[0, b]}\right\| .
$$

This number satisfies the properties

$$
\delta_{t}(N) \geq 0, \quad \delta_{t}\left(N_{1}+N_{2}\right) \leq \delta_{t}\left(N_{1}\right)+\delta_{t}\left(N_{2}\right), \quad \delta_{t}(\lambda N)=\cdot|\lambda| \delta_{t}(N)
$$

on the space $\mathcal{L}_{N}(U, U)$ of all operators of the form (22).

Theorem 7. If the operator $N$ is bounded in the space $U$, the estimate

$$
r(N) \leq \delta_{t}(N)
$$

is true.

Proof. Let $|\lambda|>\delta_{t}(N)$. We divide the rectangle $[0, a] \times[0, b]$ by straight lines $t=$ $t_{i}(i=1, \ldots, n)$ into rectangles $D_{i}=\left[t_{i-1}, t_{i}\right] \times[0, b]$ in such a way that $\left\|P_{D_{i}} N P_{D_{i}}\right\|<$ $|\lambda|$ for $i=1, \ldots, n$; this is possible by the definition of $\delta_{t}(N)$. The equation $\lambda x=N x+f$ is then replaced by a system

$$
\lambda x(t, s)=(N x)(t, s)+f(t, s) \quad\left((t, s) \in D_{i} ; i=1, \ldots, n\right) .
$$

The first equation has a unique solution on $D_{1}$, by the Banach-Caccioppoli contraction mapping theorem (observe that $|\lambda|^{-1}\left\|P_{D_{i}} N P_{D_{i}}\right\|<1$ ). Analogously, we may find a unique solution on the other sets $D_{2}, \ldots, D_{n}$. Consequently, for $|\lambda|>\delta_{t}(N)$ and $f \in U$, the equation $\lambda x=N x+f$ has a unique solution $x \in U$, and hence (24) holds 
The following example shows that one may have strict inequality in (24).

Example 2. Let $a=b=1$ and $n(t, s, \tau, \sigma)=\bar{l}(t, \tau)$, where $\bar{l}(t, \tau)$ is defined as in Example 1. Then the operator. (22) acts in $L^{\infty}$ with $r(N)=0$. On the other hand, as in Example 1 one may show that $\delta_{t}(N)=1$.

Let us say that the operator (22) has the Ando t-property if

$$
\lim _{\operatorname{mes} D \rightarrow 0}\left\|P_{D \times[0, b]} N P_{D \times[0, b]}\right\|=0
$$

Usually, this property may also be verified by means of majorant techniques. In the space $L^{p}(1 \leq p \leq \infty)$ the Andô $t$-property is satisfied for $N$, for example, if the kernel $n$ is bounded. The Andô property of $N$ implies the Andô $t$-property. In particular, a compact regular operator $N$ in a regular ideal space has the Ando $t$-property. From (23), (25) and Theorem 7 we get the following

Theorem 8. If the operator (22) has the Andôt-property, then $\delta_{t}(N)=r(N)=0$.

Consider now the operator ( 1 ) with $\Omega=[0, t] \times[0, b]$. It turns out that, under some natural assumptions, the spectral radius of the operator (1) is zero also in this case. We suppose throughout that the operator (1) is regular in some ideal space $U$. Sufficient conditions for the equality $r(L)=r(M)=0$ may be obtained from the above theorems.

The equation $x-\mu K x=f$ with $K$ given by (1) is in $U$ equivalent to each of the equations (13). The first of these equations admits a representation

$$
x(t, s)=\mu(R x)(t, s)+g(t, s)
$$

where

$$
(R x)(t, s)=\int_{0}^{t} \int_{0}^{b} r(t, s, \tau, \sigma) x(\tau, \sigma) d \tau d \sigma
$$

with

$$
\begin{aligned}
r(t, s, \tau, \sigma)= & \int_{\tau}^{t} \phi\left(t, s, \tau_{1}\right) n_{1}\left(\tau_{1}, s, \tau, \sigma\right) d \tau_{1} \\
& +\int_{0}^{s} \psi\left(t, s, \sigma_{1}\right) n_{1}\left(t, \sigma_{1}, \tau, \sigma\right) d \sigma_{1} \\
& +\int_{\tau}^{t} \int_{0}^{s} n_{2}\left(t, s, \tau_{1}, \sigma_{1}\right) n_{1}\left(\tau_{1}, \sigma_{1}, \tau, \sigma\right) d \tau_{1} d \sigma_{1} \\
& +n_{1}(t, s, \tau, \sigma)
\end{aligned}
$$


and

$$
\begin{aligned}
g(t, s)= & f(t, s) \\
& +\int_{0}^{t} \phi(t, s, \tau) f(\tau, s) d \tau \\
& +\int_{0}^{s} \psi(t, s, \sigma) f(t, \sigma) d \sigma \\
& +\int_{0}^{t} \int_{0}^{s} n_{2}(t, s, \tau, \sigma) f(\tau, \sigma) d \tau d \sigma
\end{aligned}
$$

with

$$
\begin{aligned}
& n_{1}(t, s, \tau, \sigma)=n(t, s, \tau, \sigma)+\mu l(t, s, \tau) m(\tau, s, \sigma) \times[0, s](\sigma) \\
& n_{2}(t, s, \tau, \sigma)=\psi(t, s, \sigma) \phi(t, \sigma, \tau) .
\end{aligned}
$$

Now, if the operator (27) has the Ando $t$-property, equation (26), and hence also the equation $x-\mu K x=f$, has a unique solution in $U$. Consequently, $\mu^{-1} \notin \sigma(K)$, and hence $r(K)=0$.

In this way, Theorem 3 remains valid for the operator $K$ with $\Omega=[0, t] \times[0, b]$ without any change. For the sake of completeness, we also formulate analogues to Theorems $4-6$ in this case.

Theorem 9. Let $X=L^{p}([0, a])$ and $Y=L^{p}([0, b])(1 \leq p \leq \infty)$. Suppose that the regular integral operators $L(s)(0 \leq s \leq b)$ and $M(t) \quad(0 \leq t \leq a)$ act in the spaces $X$ and $Y$, respectively, and have the Ando property. Moreover, assume that the linear integral operator (22) is regular in the space $U=L^{p}([0, a] \times[0, b])$, and that the operator (27) has the Ando t-property in $U$. Then the operator (1) satisfies $r(K)=0$.

Theorem 10. Suppose that the operators (19) act in $L^{p}([0, a])$ and $L^{p}([0, b])$, respectively, and have the Ando property. Assume that the operator (22) is regular in $U=L^{p}([0, a] \times[0, b])$ and the operator $(27)$ has the Ando $t$-property in $U$. Then the operator (1) acts in $U$ and satisfies $r(K)=0$.

Theorem 11. Let $X$ and $Y$ be ideal spaces over $[0, a]$ and $[0, b]$, respectively, and $\alpha, \beta \in L^{\infty}$ with $\alpha$ and $\beta$ given by (14). Suppose that the operators $L(s)(0 \leq s \leq b)$ and $M(t) \quad(0 \leq t \leq a)$ have the Ando property $(17) /(18)$, and that the operators

a) $\tilde{M}$ and $N$ act in $Y$ and $Y[X]$, respectively

or

b) $\tilde{L}$ and $N$ act in $X$ and $X[Y]$, respectively

and have the Ando property. Then the operator (1) acts in $Y[X]$ or $X[Y]$, respectively, and satisfies $r(K)=0$.

Of course, analogous results hold in the case $\Omega=[0, a] \times[0, s]$.

Let us indicate yet another effective way of verifying the relation $r(K)=0$ in the case $\Omega=[0, t] \times[0, s]$. Suppose that the estimates

$$
|l(t, s, \tau)| \leq a(t, \tau), \quad|m(t, s, \sigma)| \leq b(s, \sigma), \quad|n(t, s, \tau, \sigma)| \leq c a(t, \tau) b(s, \sigma)
$$


hold. If the operators

$$
(A x)(t)=\int_{0}^{t} a(t, \tau) x(\tau) d \tau \quad \text { and } \quad(B x)(s)=\int_{0}^{s} b(s, \sigma) x(\sigma) d \sigma
$$

act in two ideal spaces $X$ and $Y$, respectively, then the operator (1) is regular in $U=$ $Y[X]$ and $U=X[Y]$, and

$$
r(K) \leq r(A)+r(B)+\operatorname{cr}(A) r(B) .
$$

In particular, $r(K)=0$ if $r(A)=r(B)=0$.

\section{Operators of Volterra-Fredholm type}

We turn now to another. class of operators. For $p, q>0$ let

$$
\begin{aligned}
& \left(L_{p} x\right)(t, s)=\int_{0}^{p} l(t, s, \tau) x(\tau, s) d \tau \\
& \left(M_{q} x\right)(t, s)=\int_{0}^{q} m(t, s, \sigma) x(t, \sigma) d \sigma \\
& \left(N_{p q} x\right)(t, s)=\int_{0}^{p} \int_{0}^{q} n(t, s, \tau, \sigma) x(\tau, \sigma) d \tau d \sigma .
\end{aligned}
$$

For $p \in\{t, a\}$ and $q \in\{s, b\}$ we call then the operators

$$
\begin{aligned}
& K_{1}=L_{t}+M_{s}+N_{a b} \\
& K_{2}=L_{t}+M_{b}+N_{p q} \\
& K_{3}=L_{a}+M_{s}+N_{p q}
\end{aligned}
$$

partial integral operators of Volterra-Fredholm type. Many problems arising in continuum mechanics [1], in contact problems [2], and in other fields [4] lead to such equations. The properties of the operator (28) are essentially different from those of the operators (29) and (30). For instance, the Fredholm alternative applies to the equation $x-K_{1} x=f$ for many kernels, while it does not apply to the equations $x-K_{2} x=f$ and $x-K_{3} x=f$ even for bounded kernels.

Consider first the operator (28). Using conditions for the equalities $r(L)=r(M)=0$ and the relations (11) and (12), the equation $x-\mu K_{1} x=f$ involving a regular operator $K_{1}$ reduces to the equivalent Fredholm integral equation

$$
x(t, s)=\mu\left(R_{1} x\right)(t, s)+g(t, s)
$$


where

$$
\left(R_{1} x\right)(t, s)=\int_{0}^{a} \int_{0}^{b} r_{1}(t, s, \tau, \sigma) x(\tau, \sigma) d \tau d \sigma
$$

with

$$
\begin{aligned}
r_{1}(t, s, \tau, \sigma)= & \int_{0}^{t} \phi\left(t, s, \tau_{1}\right) n_{1}\left(\tau_{1}, s, \tau, \sigma\right) d \tau_{1} \\
& +\int_{0}^{s} \psi\left(t, s, \sigma_{1}\right) n_{1}\left(t, \sigma_{1}, \tau, \sigma\right) d \sigma_{1} \\
& +\int_{0}^{t} \int_{0}^{s} n_{2}\left(t, s, \tau_{1}, \sigma_{1}\right) n_{1}\left(\tau_{1}, \sigma_{1}, \tau, \sigma\right) d \tau_{1} d \sigma_{1} \\
& +n_{1}(t, s, \tau, \sigma)
\end{aligned}
$$

and

$$
\begin{aligned}
g(t, s)= & f(t, s) \\
& +\int_{0}^{t} \phi(t, s, \tau) f(\tau, s) d \tau \\
& +\int_{0}^{s} \psi(t, s, \sigma) f(t, \sigma) d \sigma \\
& +\int_{0}^{t} \int_{0}^{t} n_{2}(t, s, \tau, \sigma) f(\tau, \sigma) d \tau d \sigma
\end{aligned}
$$

with

$$
\begin{aligned}
& n_{1}(t, s, \tau, \sigma)=n(t, s, \tau, \sigma)+\mu l(t, s, \tau) m(\tau, s, \sigma) \chi_{[0, t]}(\tau) \chi_{[0, s]}(\sigma) \\
& n_{2}(t, s, \tau, \sigma)=\psi(t, s, \sigma) \phi(t, \sigma, \tau) .
\end{aligned}
$$

If, in addition, the operator (32) is compact, then the operator $I-\mu R_{1}$ is Fredholm of index zero. Consequently, $\lambda=\mu^{-1}$ does not belong to the Fredholm spectrum of $K_{1}$, and hence $\sigma_{e s}\left(K_{1}\right)=\{0\}$; where

$$
\sigma_{e s}\left(K_{1}\right)=\left\{\lambda \in \sigma\left(K_{1}\right): \lambda I-K_{1} \text { is not Fredholm operator of index zero }\right\} \text {. }
$$

We thus arrive at the following results.

Theorem 12. Let $X$ and $Y$ be almost perfect ideal spaces over $[0, a]$ and $[0, b]$, respectively, and $U=Y[X]$ or $U=X[Y]$. Suppose that the operator (28) is regular in $U$, and the operators $|L(s)|(0 \leq s \leq b)$ and $|M(t)|(0 \leq t \leq a)$ defined by (7) and (8), respectively, are compact in $X$ and $Y$. Finally, suppose that the resolvent kernels $\phi(t, s, \tau)$ and $\psi(t, s, \sigma)$ belong to $\mathcal{R}_{l}(U, U)$ and $\mathcal{R}_{m}(U, U)$, respectively, and the operators $N_{a b}$ and $L_{t} M_{s}$ are compact in $U$. Then $\sigma_{e s}\left(K_{1}\right)=\{0\}$. 
Theorem 13. Let $X=L^{p}([0, a])$ and $Y=L^{p}([0, b]) \quad(1 \leq p \leq \infty)$. Suppose that the integral operators $L(s)(0 \leq s \leq b)$ and $M(t) \quad(0 \leq t \leq a)$ are regular in the spaces $X$ and $Y$, respectively, and have the Ando property (17)/(18). Assume, moreover, that the operator (28) is regular in $U=L^{p}([0, a] \times[0, b])$, and the operators $N_{a b}$ and $L_{t} M$, are compact in $U$. Then $\sigma_{e s}\left(K_{1}\right)=\{0\}$.

Theorem 14. Suppose that the operators $\tilde{L}$ and $\tilde{M}$ defined by (19) act in $L^{p}([0, a])$ and $L^{p}([0, b])$, respectively, and have the Andô property. Assume that the operator $N_{a b}$ is regular in $U=L^{p}([0, a] \times[0, b])$, and the operators $N_{a b}$ and $L_{t} M_{s}$ are compact in $U$. Then the operator (28) acts in $U$ and satisfies $\sigma_{e s}\left(K_{1}\right)=\{0\}$.

Theorem 15. Let $X$ and $Y$ be almost perfect ideal spaces over $[0, a]$ and $[0, b]$, respectively. Suppose that $\alpha \in L^{\infty}$ or $\beta \in L^{\infty}$, respectively, with $\alpha$ and $\beta$ given by (14). Moreover, assume that the operators

a) $L(s) \quad(0 \leq s \leq b)$ have the Ando property (17) and $\tilde{M}$ acts in $Y$ and has the Andô property,

or

b) $M(t) \quad(0 \leq t \leq a)$ have the Andô property (18) and $\tilde{L}$ acts in $X$ and has the Andô property,

$N_{a b}$ is regular in $Y[X]$ or $X[Y]$ and $N_{a b}$ and $L_{t} M$, are compact in $Y[X]$ or $X[Y]$, respectively. Then the operator (28) acts in $Y[X]$ or $X[Y]$, respectively, and satisfies $\sigma_{e s}\left(K_{1}\right)=\{0\}$.

Theorem 14 implies, in particular, that the operator $(28)$ acts in $L^{\infty}([0, a] \times[0, b])$ with $\sigma_{e s}\left(K_{1}\right)=\{0\}$ if the kernels $l$ and $m$ in (28) are bounded and $n_{1}$ is continuous.

The operator (29) can be analyzed similarly. Using conditions for the equality $r(L)=0$ and relation (11) with $\lambda=1$, the equation $x-K_{2} x=f$ involving the regular operator $K_{2}=L_{t}+M_{b}+N_{p q}(p \in\{t, a\}, q \in\{s, b\})$ reduces to the equivalent equation

$$
x(t, s)=\left(M_{b}+\bar{N}\right) x(t, s)+g(t, s),
$$

where

$$
(\bar{N} x)(t, s)=\int_{0}^{a} \int_{0}^{b} \bar{n}(t, s, \tau, \sigma) x(\tau, \sigma) d \tau d \sigma .
$$

Here the kernel $\bar{n}$ has the form

$$
\begin{aligned}
\bar{n}(t, s, \tau, \sigma) & \\
= & \phi(t, s, \tau) m(\tau, s, \sigma) \chi_{[0, t]}(\tau) \\
& + \begin{cases}{\left[n(t, s, \tau, \sigma)+\int_{0}^{t} \phi(t, s, \xi) n(\xi, s, \tau, \sigma) d \xi\right] \chi_{[0, q]}(\sigma)} & \text { for } p=a \\
{\left[n(t, s, \tau, \sigma)+\int_{\tau}^{t} \phi(t, s, \xi) n(\xi, s, \tau, \sigma) d \xi\right] \chi_{[0, t]}(\tau) \chi_{[0, q]}(\sigma)} & \text { for } p=t\end{cases}
\end{aligned}
$$


and the function $g$ in (33) is defined by

$$
g(t, s)=f(t, s)+\int_{0}^{t} \phi(t, s, \tau) f(r, s) d \tau
$$

If the operator (34) is compact, then the operator $I-K_{2}$ is Fredholm of index zero if and only if the operator $I-M_{b}$ is so: In particular, in the case $m(t, s, \sigma) \equiv m(s, \sigma)$ the operator $I-K_{2}$ is Fredholm of index zero if and only if the operator $I-M_{b}$ is invertible.

The same approach works for the operator (30). We do not formulate the corresponding results which are similar to Theorems $12-15$.

\section{References}

[1] Aleksandrov, V. M. and E. V. Kovalenko: On a class of integral equations of mixed problems arising in the mechanics of continuous media (in Russian). Dokl. Akad. Nauk SSSR 252 (1980), 324 - 328; Engl. transl.: Soviet Phys. Dokl. 25 (1980), 354 - 356.

[2] Aleksandrov, V. M. and E. V. Kovalenko: Problems Arising in the Mechanics of Continuous Media with Mixed Boundary Conditions (in Russian). Moscow: Nauka 1986.

[3] Bukhvalov, A. V.: On spaces with mixed norm (in Russian). Vestnik Leningr. Gos. Univ. 19 (1973), 5 - 12.

[4] Gabov, S. A. and A. G. Sveshnikov: Linear Problems of Non-Stationary Inner Waves (in Russian). Moscow: Nauka 1990.

[5] Kalitvin, A. S. and P. P. Zabrejko: On the theory of partial integral operators. J. Int. Equ. Appl. 3 (1991), $351-382$.

[6] Krasnosel'skij, M. A., Zabrejko, P. P., Pustyl'nik, E. I. and P. E. Sobolevskij: Integral Operators in Spaces of Summable Functions. Lejden: Noordhoff 1976.

[7] Väth, M.: Ideal Spaces. Berlin: Springer 1997.

[8] Väth, M.: Abstract Volterra equations of the second kind. J. Int. Equ. Appl. (to appear).

[9] Zabrejko, P. P.: Nonlinear integral operators (in Russian). Trudy Sem. Funk. Anal. Voronezh 8 (1966), 1 - 148.

[10] Zabrejko, P. P.: On Volterra integral operators (in Russian). Lit. Mat. Sbornik 7 (1967), $281-287$.

[11] Zabrejko, P. P.: Ideal function spaces (in Russian). Vestnik Jarosl. Gos. Univ. 8 (1974), $12-52$.

[12] Zabrejko, P. P. and A. N. Lomakovich: Volterra integral operators in spaces of functions of two variables (in Russian). Ukrain. Mat. Zh. 42 (1990), 1187 - 1191; Engl. transl.: Ukrain. Math. J. 42 (1990), 1055 - 1058.

Received 17.08.1997; in revised form 07.01.1998 